\title{
BMJ Open The Esophageal Pressure-Guided Ventilation 2 (EPVent2) trial protocol: a multicentre, randomised clinical trial of mechanical ventilation guided by transpulmonary pressure
}

\author{
Emily Fish, ${ }^{1}$ Victor Novack, ${ }^{2}$ Valerie M Banner-Goodspeed, ${ }^{1,3}$ Todd Sarge, ${ }^{1}$ \\ Stephen Loring, ${ }^{1}$ Daniel Talmor ${ }^{1}$
}

To cite: Fish E, Novack V, Banner-Goodspeed VM, et al. The Esophageal PressureGuided Ventilation 2 (EPVent2) trial protocol: a multicentre, randomised clinical trial of mechanical ventilation guided by transpulmonary pressure. BMJ Open 2014;4:e006356. doi:10.1136/bmjopen-2014006356

- Prepublication history for this paper is available online To view these files please visit the journal online (http://dx.doi.org/10.1136/ bmjopen-2014-006356).

Received 14 August 2014 Accepted 22 August 2014

CrossMark

${ }^{1}$ Department of Anesthesia, Critical Care, \& Pain Medicine, Beth Israel Deaconess Medical Center and Harvard Medical School, Boston, Massachusetts, USA ${ }^{2}$ Soroka University Medical Center, Rager Boulevard, Beer-Sheva, Israel

${ }^{3}$ Department of Anesthesia, Critical Care, \& Pain Medicine, Beth Israel Deaconess Medical Center, Boston, Massachusetts, USA

Correspondence to

Dr Daniel Talmor;

dtalmor@bidmc.harvard.edu

\section{ABSTRACT}

Introduction: Optimal ventilator management for patients with acute respiratory distress syndrome (ARDS) remains uncertain. Lower tidal volume ventilation appears to be beneficial, but optimal management of positive end-expiratory pressure (PEEP) remains unclear. The Esophageal PressureGuided Ventilation 2 Trial (EPVent2) aims to examine the impact of mechanical ventilation directed at maintaining a positive transpulmonary pressure $\left(\mathrm{P}_{\mathrm{TP}}\right)$ in patients with moderate-to-severe ARDS.

Methods and analysis: EPVent2 is a multicentre, prospective, randomised, phase II clinical trial testing the hypothesis that the use of a $\mathrm{P}_{\mathrm{TP}}$-guided ventilation strategy will lead to improvement in composite outcomes of mortality and time off the ventilator at 28 days as compared with a high-PEEP control. This study will enrol 200 study participants from 11 hospitals across North America. The trial will utilise a primary composite end point that incorporates death and days off the ventilator at 28 days to test the primary hypothesis that adjusting ventilator pressure to achieve positive $P_{\text {TP }}$ values will result in improved mortality and ventilator-free days.

Ethics and dissemination: Safety oversight will be under the direction of an independent Data and Safety Monitoring Board (DSMB). Approval of the protocol was obtained from the DSMB prior to enrolling the first study participant. Approvals of the protocol as well as informed consent documents were also obtained from the Institutional Review Board of each participating institution prior to enrolling study participants at each respective site. The findings of this investigation, as well as associated ancillary studies, will be disseminated in the form of oral and abstract presentations at major national and international medical specialty meetings. The primary objective and other significant findings will also be presented in manuscript form. All final, published manuscripts resulting from this protocol will be submitted to PubMed Central in accordance with the National Institute of Health Public Access Policy. Trial registration number: ClinicalTrials.gov under number NCT01681225.

\section{INTRODUCTION}

Optimal ventilator management for patients with acute respiratory distress syndrome (ARDS) remains uncertain. Lower tidal volume ventilation appears to be beneficial, ${ }^{1}$ but optimal management of positive end-expiratory pressure (PEEP) remains unclear. Higher levels of PEEP have been shown to be protective in numerous animal experiments but have only shown equivocal benefit in human clinical trials. ${ }^{2}$ Recently, however, an individual patient meta-analysis combining data from these trials suggested that higher PEEP, when added to use of lower tidal volumes, results in an adjusted risk ratio of $0.90 \quad(95 \%$ CI 0.81 to 1.00 ; $\mathrm{p}=0.049$ ) in patients with moderate-to-severe ARDS. $^{3}$ Thus, incremental improvements offer the potential for additional mortality reduction, and the central question remains how best to optimise PEEP for an individual patient.

Lung inflation reflects transpulmonary pressure $\left(\mathrm{P}_{\mathrm{TP}}\right)$, the difference between pressures at the airway opening and the pleural space $\left(\mathrm{P}_{\mathrm{TP}}=\right.$ airway pressure-pleural pressure). Clinicians routinely manage mechanical ventilation by controlling pressures measured at the airway. This would be a satisfactory strategy if pleural pressure values were predictable or restricted to a narrow range. However, there is evidence that pleural pressure varies widely and unpredictably among patients with ARDS, likely due to factors such as obesity, abdominal fluid accumulation and oedema, which influence the mechanical behaviour of the chest wall and diaphragm. ${ }^{4}$ This wide range of pleural pressure among individuals could significantly affect lung inflation produced by mechanical 
ventilation. For example, a relatively high level of PEEP of $18 \mathrm{~cm} \mathrm{H} \mathrm{H}_{2} \mathrm{O}$ could be too low in a patient with a pleural pressure of $20 \mathrm{~cm} \mathrm{H}_{2} \mathrm{O}$, allowing collapse of some airspaces with each expiration and leading to atelectrauma, ${ }^{5}$ or too high in a patient with a pleural pressure of $5 \mathrm{~cm} \mathrm{H}_{2} \mathrm{O}$, causing haemodynamic compromise, increased dead space ventilation and overdistension of the lungs at end-inflation. It is possible that the inconsistency in the results of clinical trials of PEEP $^{6} 7$ reflects a failure to individualise ventilator settings for pleural pressure in each patient. ${ }^{8}$

In a single centre, phase I randomised controlled trial, mechanical ventilation directed by esophageal pressure was compared with ventilation using the ARDSNet protocol. In this study, nearly all patients who were managed using the esophageal pressure measurements required adjustment upwards of their set PEEP level to maintain a transpulmonary pressure of greater than zero. The ratio of blood oxygen tension to inspired oxygen fraction $\left(\mathrm{PaO}_{2}\right.$, partial pressure of oxygen dissolved in arterial blood $/ \mathrm{FiO}_{2}$, fraction of inspired oxygen; $\mathrm{PaO}_{2} / \mathrm{FiO}_{2}$ ) at $72 \mathrm{~h}$ was higher in the intervention (EPVent) group than controls, and respiratory system compliance was significantly improved. This trial showed a trend towards reduced mortality that was maintained at 6 months. ${ }^{9}$

To further explore the use of $\mathrm{P}_{\mathrm{TP}}$ values to guide ventilation in patients with ARDS, our group, with the support of the National Heart, Lung and Blood Institute (NHLBI), has designed the EPVent2 study, a randomised clinical trial that aims to examine the impact of mechanical ventilation directed at maintaining a positive $\mathrm{P}_{\mathrm{TP}}$ in patients with moderate-to-severe ARDS. This paper describes the study procedures and planned analyses for this clinical trial. The trial is registered on ClinicalTrials.gov under number NCT01681225.

\section{METHODS AND ANALYSIS}

\section{Administrative structure}

This study is under the auspices of the NHLBI (Grant Number U01-HL108724). The Medical Coordinating

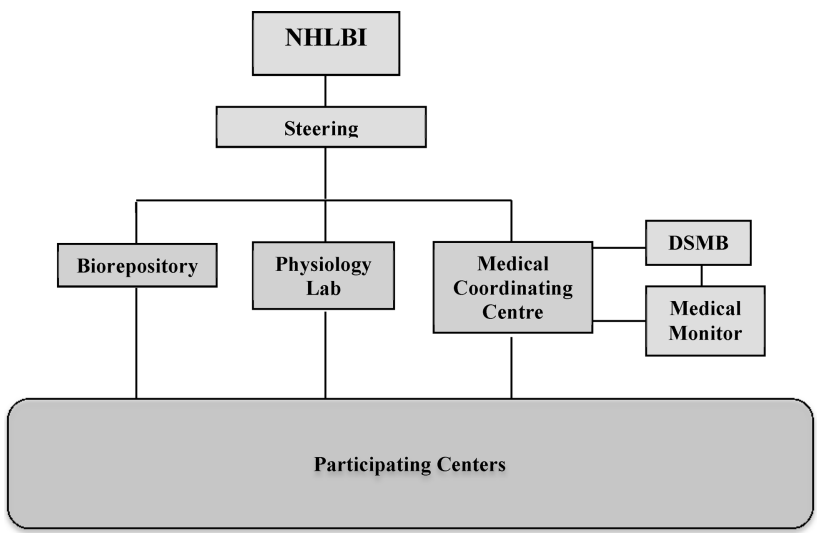

Figure 1 Administrative structure (NHLBI, National Heart, Lung and Blood Institute; DSMB, Data and Safety Monitoring Board).
Center (MCC) at Beth Israel Deaconess Medical Center (BIDMC) is responsible for study conduct, safety monitoring, personnel training, data management and randomisation. The Physiology Core Laboratory (PCL), also at BIDMC, analyses waveforms collected on each patient and provides specialised equipment and software training to study centres. The biospecimen repository at the Harvard School of Public Health in Boston, Massachusetts, USA, is responsible for performing primary analyses on all specimens, as well as storing remaining specimens from patients who have given separate consent in a biorepository. The EPVent2 Steering Committee consists of the head of the MCC and the PCL, a scientific advisor and the principal investigator (PI) from each recruiting centre. This committee collaboratively oversees all aspects of the study design and the protocol implementation (figure 1).

\section{Study design}

To test the hypothesis that adjusting ventilator pressure to achieve positive $\mathrm{P}_{\mathrm{TP}}$ values will lead to improvement in composite outcomes of mortality and time off the ventilator at 28 days, the EPVent2 group has designed a multicentre, prospective, randomised, phase II clinical trial. Overall study flow is presented in figure 2.

\section{Development of the EPVent2 trial protocol}

There are several key differences between the EPVent1 trial and the EPVent2 trial. In the current trial, we limit enrolment to patients with moderate or severe ARDS (P/F of 200 or below). We also have changed the control group protocol to a higher PEEP approach. The duration of our intervention has been extended from 3 to 28 days for the EPVent2 trial. Finally, our primary end point for the current trial is a novel composite measure that combines ventilator-free days and mortality at 28 days, whereas in the EPVent1 trial with fewer participants we studied the improvement in oxygenation at $72 \mathrm{~h}$. Table 1 summarises the main differences between the two protocols.

\section{Restriction of enrolled patients to moderate-to-severe ARDS}

In this trial we elected to restrict enrolment to patients with moderate-to-severe ARDS with $\mathrm{P} / \mathrm{F}<200$. This decision was primarily based on a meta-analysis published by Briel et $a l^{3}{ }^{3}$ which showed higher levels of PEEP to be associated with increased survival among the subset of patients with what is now defined as moderate-to-severe ARDS $(\mathrm{P} / \mathrm{F}<200)$. Given that the $\mathrm{P}_{\mathrm{TP}}$-guided strategy typically leads to ventilation using higher levels of PEEP, we felt limitation to patients with moderate-to-severe ARDS to be both clinically and scientifically appropriate. Additionally, this restriction may enhance the difference between the EPVent group and the control group insofar as sicker patients stand to gain the most from individualised treatment. 
Figure 2 Overview of study design (ARDS, acute respiratory distress syndrome; EPVent, Esophageal Pressure-Guided Ventilation).

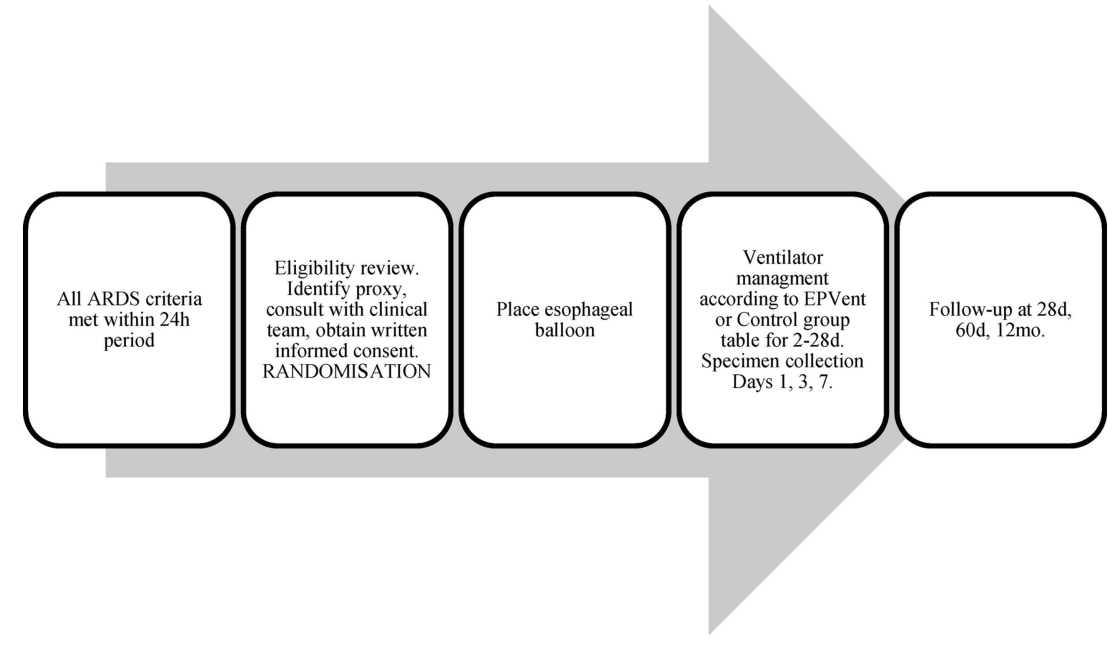

\section{Control arm strategy}

In this trial we opted to use a high-PEEP control arm as opposed to the ARDSNet ventilation guidelines used in our pilot study. The rationale for this modification is based on three key points. First, given our enrolment restriction to patients with moderate-to-severe ARDS, we did not feel comfortable utilising a low-PEEP strategy for the control arm based on the findings by Briel $e t a l$ discussed previously. Additionally, our phase I study had already shown benefit of a $\mathrm{P}_{\mathrm{TP}}$-guided strategy over a low-PEEP strategy. ${ }^{9}$ Finally, we wanted to ensure that the

Table 1 Comparison of EPVent1 phase 1 and EPVent2 phase 2 trials

\begin{tabular}{|c|c|c|}
\hline Factor & EPVent1 & EPVent2 \\
\hline $\begin{array}{l}\text { Number of } \\
\text { participants }\end{array}$ & 61 actual & 200 planned \\
\hline $\begin{array}{l}\text { Number of } \\
\text { centres }\end{array}$ & 1 & 11 planned \\
\hline $\begin{array}{l}\text { Control group } \\
\text { strategy }\end{array}$ & $\begin{array}{l}\text { ARDSNet } \\
\text { study (low tidal } \\
\text { volume) }\end{array}$ & $\begin{array}{l}\text { OSCILLATE study } \\
\text { control group } \\
\text { (high-PEEP) }\end{array}$ \\
\hline Funding & Institutional & NIH/NHLBI \\
\hline $\begin{array}{l}\text { ARDS } \\
\text { severity }\end{array}$ & $P / F<300$ & $\mathrm{P} / \mathrm{F}<200$ \\
\hline Outcomes & $\begin{array}{l}\mathrm{PaO}_{2}: \mathrm{FiO}_{2} \text { at } \\
72 \mathrm{~h}\end{array}$ & $\begin{array}{l}\text { Composite measure of } \\
\text { mortality and } \\
\text { ventilator-free days at } \\
28 \text { days }\end{array}$ \\
\hline $\begin{array}{l}\text { Duration of } \\
\text { intervention }\end{array}$ & 3 days & 28 days \\
\hline $\begin{array}{l}\text { Additional } \\
\text { studies }\end{array}$ & $\begin{array}{l}\text { Biomarker } \\
\text { analysis }\end{array}$ & Biomarker analysis \\
\hline & & $\begin{array}{l}\text { Echocardiography } \\
\text { CT }\end{array}$ \\
\hline \multicolumn{3}{|c|}{$\begin{array}{l}\text { ARDS, acute respiratory distress syndrome; EPVent, Esophageal } \\
\text { Pressure-Guided Ventilation; } \mathrm{FiO}_{2} \text {, fraction of inspired oxygen; } \\
\mathrm{NHLBI} \text {, National Heart, Lung and Blood Institute; } \mathrm{NIH} \text {, National } \\
\text { Institutes of Health; } \mathrm{PaO}_{2} \text {, partial pressure of oxygen dissolved in } \\
\text { arterial blood; PEEP, positive end-expiratory pressure. }\end{array}$} \\
\hline
\end{tabular}

appropriate comparison was being made between ventilation strategies to determine whether empirically raising PEEP (through a high-PEEP control arm) will result in the same outcomes as those achieved using a $\mathrm{P}_{\mathrm{TP}}$-guided ventilation strategy. We therefore adopted a high-PEEP algorithm previously used in the OSCILLATE trial for the control group arm. ${ }^{10}$

\section{Box 1 Esophageal Pressure-Guided Ventilation 2} (EPVent2) study exclusion criteria

- Received mechanical ventilation more than $96 \mathrm{~h}$.

- Recently treated for bleeding varices, stricture, haematemesis, esophageal trauma, recent esophageal surgery or other contraindication for nasogastric tube placement.

- Severe coagulopathy (platelet count $<5000 / \mu \mathrm{L}$ or international normalised ratio $>4$ ).

- History of lung or liver transplantation.

- Elevated intracranial pressure or conditions where hypercapnia-induced elevations in intracranial pressure should be avoided (including intracranial bleeding, cerebral contusion, cerebral oedema, mass effect (midline shift on CT scan), flat EEG for $\geq 2 \mathrm{~h}$ ).

- Evidence of active air leak from the lung (including bronchopleural fistula, pneumothorax, pneumomediastinum or air leak from existing chest tube).

- Participation in other intervention trials for acute respiratory distress syndrome, or sepsis within the past 30 days.

- Neuromuscular disease that impairs ability to ventilate spontaneously (including C5 or higher spinal cord injury, amyotrophic lateral sclerosis, Guillain-Barre syndrome or myasthenia gravis).

- Severe chronic liver disease (Child-Pugh Score $\geq 12$ ).

- Patients not committed to full support.

- Treating clinician refusal, or unwillingness to commit to controlled ventilation for at least $24 \mathrm{~h}$.

- Inability to get informed consent from the patient or surrogate.

- Use of rescue therapies prior to enrolment (including nitric oxide, ECMO, prone positioning or high frequency oscillation), unless therapies were used as the initial mode of ventilation. 


\section{Study population}

Adult patients aged 16 years and older, admitted to the intensive care unit (ICU) with moderate-to-severe ARDS, will be enrolled. The Berlin Conference definition will be used to identify patients with moderate-to-severe ARDS, including (A) hypoxaemic respiratory failure with $\mathrm{PaO}_{2}$ / $\mathrm{FiO}_{2}$ ratio $<200 \mathrm{~mm} \mathrm{Hg}$, (B) bilateral alveolar infiltrates on chest X-ray not present for more than 7 days, (C) respiratory failure not fully explained by cardiac failure or fluid overload and (D) intubation on controlled ventilation and receiving PEEP $\geq 5 \mathrm{~cm} \mathrm{H}_{2} \mathrm{O}$. Patients with duration of ARDS $\geq 36 \mathrm{~h}$ or duration of mechanical ventilation $\geq 96 \mathrm{~h}$ will be excluded. A full list of exclusion criteria can be seen in box 1 .

Patients will be recruited from approximately 11 clinical sites in North America with experience in the identification and management of ARDS. A full list of the participating institutions is displayed in box 2. The resulting study population is expected to be representative of the North American adult acute care hospital population.

Study coordinators at each site will visit the ICUs daily to identify potential candidates for enrolment. Screening logs will be maintained at each site and tracked using an electronic screening tool (Studymaker. com). Once a patient is deemed eligible for the study, the designated substitute decision maker will be approached by a study investigator to give informed consent. Eligible patients will be enrolled, randomised and initial ventilator adjustments made within $36 \mathrm{~h}$ of the time the last ARDS criterion was met.

\section{Randomisation and blinding}

After enrolment, patients will be randomised to either the EPVent group or the control group using a block randomisation scheme at a 1:1 ratio. Randomisation will be stratified by enrolment site. Assignment schedules will be generated for each clinical site, and each patient will be assigned using a web-based system (Studymaker. com) managed by the MCC.

Box 2 Esophageal Pressure-Guided Ventilation 2 (EPVent2) study participating centres

Beth Israel Deaconess Medical Center, Boston, Massachusetts, USA.

- Laval University, Quebec City, Quebec, Canada.

- Massachusetts General Hospital, Boston, Massachusetts, USA.

- Montefiore Medical Center, Bronx, New York, USA.

- Orlando Health Systems, Orlando, Florida, USA.

- St. Joseph's Hospital, Hamilton, Ontario, Canada.

- Toronto General Hospital, Toronto, Ontario, Canada.

- University of Cincinnati, Cincinnati, Ohio, USA.

- University of Massachusetts Memorial Medical Center, Worcester, Massachusetts, USA.

- University of Michigan, Ann Arbor, Massachusetts, USA.

- Vancouver General Hospital, Vancouver, British Columbia, Canada.
All patients must be enrolled, randomised and initial ventilator adjustments made within $36 \mathrm{~h}$ of the time the last inclusion criterion was met. The last inclusion criterion may be met at either the network hospital or a referring hospital.

Investigators will remain blinded to the results of the study until study completion. The patient safety monitor, unblinded statistician and Data and Safety Monitoring Board (DSMB) will have full access to unblinded data throughout the study.

\section{Interventions}

Ventilation strategy

Following randomisation to either the intervention (EPVent) or the control group, esophageal balloon catheters will be placed in all patients. The following variables will then be measured prior to initiation of study ventilation: airway pressure, flow, esophageal pressure, $\mathrm{P}_{\mathrm{TP}}$ and expired partial pressure of carbon dioxide to allow calculation of dead space.

Once adequately sedated, all participants will have an initial alveolar recruitment manoeuvre for $30 \mathrm{~s}$ at $35 \mathrm{~cm} \mathrm{H}_{2} \mathrm{O}$ to standardise the lung volume history. The ventilation mode will be set to either pressure control or volume control ventilation. Tidal volume $(\mathrm{Vt})$ will be set to $6 \mathrm{~mL} / \mathrm{kg}$ and monitored to remain within a working range of $4-6 \mathrm{~mL} / \mathrm{kg}$. Target $\mathrm{PaO}_{2}$ will be $55-80 \mathrm{~mm} \mathrm{Hg}$, and target pulse oximeter saturation $\left(\mathrm{SpO}_{2}\right)$ 88-93\%.

\section{EPVent group}

For patients randomised to the EPVent group, the overall goal is to employ an open-lung strategy that includes low tidal volumes and maintenance of a positive $\mathrm{P}_{T P}$ at end-expiration. After measuring the $\mathrm{P}_{\mathrm{TP}}$ during an expiratory hold $\left(\mathrm{Ptp}_{\exp }\right)$, the PEEP and $\mathrm{FiO}_{2}$ will be adjusted to achieve a $\mathrm{Ptp}_{\exp }$ and $\mathrm{FiO}_{2}$ combination shown in one of the columns of table 2. Ventilator adjustments will then be made as required by blood gas values or oxygen saturation by moving right or left, one table column at a time.

Once a patient has reached step 2 or 3 of table 2 and has been on the EPVent protocol for at least $48 \mathrm{~h}$, they may be transitioned to the conventional $\mathrm{PEEP} / \mathrm{FiO}_{2}$ table and begin weaning (table 3 ).

\section{Control group}

For patients randomised to the control group, the overall goal is to also employ an open-lung strategy using low tidal volumes and an alternative high-PEEP strategy. The initial ventilator settings will be chosen from the control grid, shown in table 4 before measuring the esophageal pressure.

Choosing the settings from the table prior to measuring esophageal pressure will help reduce possible influence of knowledge of pressure measurements on the control patient's ventilator settings. The control group PEEP and $\mathrm{FiO}_{2}$ will be managed without reference to the esophageal pressure measurements, moving right or left on the table as required by blood gas values or 
Table 2 Oxygenation management table-EPVent group

\begin{tabular}{|c|c|c|c|c|c|c|c|c|c|c|c|c|c|}
\hline Step & 1 & 2 & 3 & 4 & 5 & 6 & 7 & 8 & 9 & 10 & 11 & 12 & 13 \\
\hline $\mathrm{FiO}_{2}$ & 0.3 & 0.4 & 0.5 & 0.5 & 0.6 & 0.6 & 0.7 & 0.7 & 0.8 & 0.8 & 0.9 & 0.9 & 1.0 \\
\hline Ptp & 0 & 0 & 0 & 2 & 2 & 3 & 3 & 4 & 4 & 5 & 5 & 6 & 6 \\
\hline
\end{tabular}

EPVent, Esophageal Pressure-Guided Ventilation; $\mathrm{FiO}_{2}$, fraction of inspired oxygen; Ptpexp, end-expiratory transpulmonary pressure.

oxygen saturation. Goal plateau pressure in the control group is $\leq 35 \mathrm{~cm} \mathrm{H} \mathrm{H}_{2} \mathrm{O}$. If plateau pressure exceeds $35 \mathrm{~cm} \mathrm{H}{ }_{2} \mathrm{O}$, tidal volume will be decreased to 5 or $4 \mathrm{~mL} / \mathrm{kg}$ PBW as necessary.

Protocol ventilation will be followed in both groups until the patient is capable of unassisted breathing for a period of $48 \mathrm{~h}$, dies, is discharged or otherwise withdrawn from the study, for a maximum of 28 days. Weaning may be initiated after the following criteria are met: (A) $\mathrm{FiO}_{2} \leq 0.40$, (B) $\mathrm{PEEP} \leq 10 \mathrm{~cm} \mathrm{H}_{2} \mathrm{O}$, (C) systolic arterial blood pressure is $\geq 80 \mathrm{~mm} \mathrm{Hg}$ without escalating vasopressor requirements or haemodynamic instability and (D) the patient is able to make breathing efforts to trigger the ventilator for pressure support ventilation. Weaning procedures will be common to both groups and protocol driven.

Patients will be considered to have completed the study ventilator procedures if any of the following conditions occur: (A) death, (B) hospital discharge, (C) patient or proxy withdraws consent, (D) clinician or researcher determines that the trial is no longer in the patient's best interest or (E) completion of a period of $48 \mathrm{~h}$ of unassisted breathing. Any additional requirements after this point for positive pressure ventilation will be addressed by the team caring for the patient.

\section{Co-interventions}

Patients in both groups will be managed according to existing care protocols at the study sites. These include sedation, glucose control, sepsis management and infection control protocols. These existing protocols at each site have been reviewed by the PIs and found to be in line with current practice.

\section{Blood sampling}

Blood samples will be obtained at baseline (after randomisation and before initiation of study intervention) on day 3 of study and on day 7 of study. Sample biomarkers of interest are listed in table 5. Patients will have the

$\begin{aligned} & \text { Table } 3 \\
& \text { Transitioning EPVent group to control settings } \\
& \text { for weaning }\end{aligned}$
\begin{tabular}{lllllllll}
\hline Step & $\mathbf{1}$ & $\mathbf{2}$ & $\mathbf{3}$ & $\mathbf{3}$ & $\mathbf{5}$ & $\mathbf{6}$ & \\
\hline $\mathrm{FiO}_{2}$ & 0.3 & 0.3 & 0.3 & 0.4 & 0.4 & 0.4 & Return \\
$\mathrm{PEEP}$ & 5 & 8 & 10 & 10 & 12 & 14 & $\begin{array}{l}\text { patient to the } \\
\text { EPVent } \\
\end{array}$ \\
& & & & & & & $\begin{array}{l}\text { protocol } \\
\text { settings }\end{array}$ \\
\hline
\end{tabular}

EPVent, Esophageal Pressure-Guided Ventilation; $\mathrm{FiO}_{2}$, fraction of inspired oxygen; PEEP, positive end-expiratory pressure. option of consenting to have their blood stored in a biorepository for future investigations, including genetic testing. For patients who grant specific consent, any blood left over after biomarker analyses will be stored indefinitely in the biorepository at the Harvard School of Public Health.

\section{Echocardiography}

At select centres, a complete transesophageal echocardiogram will be performed on patients in both groups at the following time points: (1) prior to initiation of study procedures and (2) $24 \mathrm{~h}$ after enrolment. These examinations will be performed only at participating sites.

\section{Data collection}

Baseline demographics and clinical characteristics such as age, sex, race, comorbidities, ARDS risk factors, Sequential Organ Failure Assessment (SOFA) score, concomitant medications (eg, aspirin, steroids, statin) and co-interventions (eg, prone positioning, nitric oxide therapy, continuous renal replacement therapy) will be recorded. Additional variables collected throughout study enrolment will include vital signs and laboratory values obtained during the course of routine clinical care, sedative and paralytic administration, vasopressor requirements, use of rescue therapies and chest tube placement. The investigation will utilise the REDCap system for data management and storage. This secure, web-based system provides robust data validation routines, custom reporting and straightforward integration with statistical software packages such as Statistical Analysis Software (SAS).

\section{Outcomes}

\section{Clinical outcomes}

The trial will utilise a primary composite end point that incorporates death and days off the ventilator at 28 days in such a manner that death constitutes a more serious outcome. Every participant in the treatment group is compared with every participant in the control group and assigned one number resulting from each comparison as shown in table 6. As mortality outcome is clinically more important, death takes precedence over days off the ventilator. These scores are summed to obtain a cumulative score for each participant. The cumulative scores are then tallied for each treatment group to form a test statistic by the Mann-Whitney technique.

Secondary clinical outcome assessments will include requirement for rescue therapies, length of mechanical 
Table 4 Oxygenation management table-control group

\begin{tabular}{|c|c|c|c|c|c|c|c|c|c|c|c|c|c|c|c|c|c|}
\hline Step & 1 & 2 & 3 & 4 & 5 & 6 & 7 & 8 & 9 & 10 & 11 & 12 & 13 & 14 & 15 & 16 & 17 \\
\hline $\mathrm{FiO}_{2}$ & 0.3 & 0.3 & 0.3 & 0.4 & 0.4 & 0.4 & 0.4 & 0.4 & 0.5 & 0.5 & 0.6 & 0.7 & 0.8 & 0.8 & 0.9 & 1.0 & 1.0 \\
\hline PEEP & 5 & 8 & 10 & 10 & 12 & 14 & 16 & 18 & 18 & 20 & 20 & 20 & 20 & 22 & 22 & 22 & 24 \\
\hline
\end{tabular}

$\mathrm{FiO}_{2}$, fraction of inspired oxygen; PEEP, positive end-expiratory pressure.

ventilation and length of stay in ICU and hospital. Hospital survivors will undergo a brief follow-up phone survey to assess survival, functional status (Barthel Index), health-related quality of life (SF-12) and frailty (VES-13) at 12 months after enrolment.

\section{Mechanistic outcomes}

Secondary analyses will include evaluations of the clinical efficacy of adjusting ventilator pressure to achieve appropriate Ptp values as predicted by identifiable features of respiratory mechanics. Specifically, the study will examine correlations among esophageal pressure, gastric pressure and bladder pressure, as well as the correlation between esophageal pressure and plateau pressure, a known predictor of mortality in ARDS. In addition, the study will examine the relationship between $\mathrm{P}_{\mathrm{TP}}$-guided ventilation and the levels of biomarkers previously associated with ventilator-induced lung injury and with poor outcomes in ALI (table 5).

\section{Sample size estimation}

To adequately address our primary hypothesis, the sample size is estimated to be 100 participants per group (200 total). Sample size calculations were performed by simulating 1000 samples on SAS software with basic distribution features of our pilot study. The following assumptions were made:

1. We assume that 28-day mortality will be $30 \%$ and $20 \%$ in control and EPVent groups, respectively (conservative estimate as compared with $38.7 \%$ in the conventional treatment and $16.7 \%$ in the EPVent treatment observed in the phase I study).

2. The distribution of days free from ventilation followed the properties of the pilot study and was assigned in two stages. First, the proportion of patients with 0 days free from ventilation was assigned (as $10 \%$ in the conventional treatment and $15 \%$ in the EPVent treatment, reflecting the higher percentage of alive but still ventilated patients at day 28 in the EPVent group). Second, the remaining values were assigned normal values with mean equal to 13.0 and 15.0 days in the conventional and EPVent groups, respectively (SD 6.5 days in both groups).

\section{Statistical methods}

Primary analyses

Primary analyses will use the set of all randomised participants (intention-to-treat (ITT) sample). We anticipate compliance to be high, but it is possible that patients in the EPVent arm may not initiate the EPVent protocol due to difficulty placing the esophageal balloon. In addition, patients who die in the first $24 \mathrm{~h}$ might be too sick to gain from the presumed benefit of EPVent-based ventilation. Therefore, a second analysis set, per protocol (PP sample) will use participants who (A) survived $24 \mathrm{~h}$ from randomisation; (B) underwent the assigned intervention (ie, the control or intervention ventilation protocol was initiated and successfully implemented for at least $24 \mathrm{~h}$ ) and $(\mathrm{C})$ have data on day 28 assessment available. The results of analyses using PP will be compared with results using the full set of all randomised participants (ITT analyses) to test sensitivity of primary findings.

\section{Missing data}

We anticipate that the proportion of participants with missing primary evaluation will be small $(<1 \%)$. For the primary analysis, we will assign death to all patients lost to follow-up prior to the 28 days. Secondary sensitivity analysis will consider these patients alive and their

Table 5 Biomarkers of interest

\section{Plasma biomarker}

Surfactant protein-D (SP-D) $)^{11-13}$

Receptor for advanced glycation end products (RAGE) ${ }^{14-16}$ Intercellular adhesion molecule-1 (ICAM-1) $)^{17-20}$

Interleukin-6 (IL-6) ${ }^{13} 21-23$

IL-8 $8^{13} 16$ 20-23

Plasminogen activator inhibitor-1 (PAl-1) ${ }^{13} 20$ 24-28

Von Willebrand factor (VWF) 262930

Protein C (PROTC) 1320242831

Tumour necrosis factor receptor (TNFR) ${ }^{13} 3233$

\section{Importance in ALI/ARDS development}

Reflect injury and $\uparrow$ permeability of alveolar epithelium Reflects endothelial activation and injury Reflects endothelial activation and injury Inflammation

Inflammation

Activation of coagulation and inhibition of fibrinolysis Reflects endothelial activation and injury Activation of coagulation and inhibition of fibrinolysis Inflammation 
Table 6 Assignment of scores for primary outcome

\begin{tabular}{|c|c|c|c|c|c|}
\hline \multirow[b]{2}{*}{ Scenario } & \multicolumn{2}{|l|}{ Mortality } & \multirow{2}{*}{$\begin{array}{l}\text { Days off ventilator } \\
\text { Control versus EPVent }\end{array}$} & \multicolumn{2}{|l|}{ Score } \\
\hline & Control & EPVent & & Control & EPVent \\
\hline 1 & No & No & $>$ & +1 & -1 \\
\hline 2 & No & No & $<$ & -1 & +1 \\
\hline 3 & No & No & $=$ & 0 & 0 \\
\hline 4 & Yes & Yes & {$[>,<,=][>,<,=][>,<,=]$} & 0 & 0 \\
\hline 5 & No & Yes & & +1 & -1 \\
\hline 6 & Yes & No & & -1 & +1 \\
\hline
\end{tabular}

ventilation status assessment will be based on the last available observation.

\section{Interim analyses}

An interim analysis is planned at $50 \%$ of enrolment. We do not propose an early stopping rule for efficacy or futility as this phase II trial is unlikely to result in a benefit with CIs narrow enough to provide compelling evidence to change practice. However, the DSMB may stop the trial for safety at any time. The DSMB operations (reporting structure, specific stopping rules, etc) are outlined in a DSMB charter signed and approved by all members.

Clinical events, including death, are evaluated for expectedness based on the patient's condition and on possible relationship to trial participation. Events and classifications are tabulated and summarised by treatment group. Also, all serious and unexpected adverse events will be summarised by the treatment group. Fisher's exact test will be used to estimate treatment differences in the incidence of each specified adverse event. No adjustments will be made for multiple hypothesis evaluations of safety end points.

\section{Safety, ethics and dissemination}

\section{Adverse outcomes}

Safety data including adverse events such as esophageal injury, loss of airway related to balloon placement, abnormal air collection, barotrauma, pneumothorax, unexpected haemodynamic instability and mortality will be recorded. Adverse events will be defined as 'unexpected', 'expected', graded by severity (mild-moderate-severe), and classified as 'serious' or 'non-serious'. As our patient population is by definition critically ill, it is expected that patients will have a number of unrelated adverse health events during the course of their hospital stay. Therefore, we will limit the scope of our adverse event monitoring and recording to the following:

1. Serious adverse events (SAEs) will be defined as:

- Death, believed to be related to the study medication or procedures, or a death that is unexpected considering the acuity of a patient.

- A life-threatening experience believed to be related to the study medication or procedures.
- Persistent or significant disability or incapacity that is of greater frequency or severity than what would be normally expected in the course of critical illness.

- An event that jeopardises the human subject and may require medical or surgical treatment to prevent one of the preceding outcomes and is not expected in the course of critical illness.

2. Adverse events possibly related to esophageal balloon placement or mechanical ventilation will be defined as:

- Barotrauma;

- Pneumothorax;

- Other abnormal air collection (eg, development of new bronchopleural fistula);

- Loss of airway related to device;

- Haemodynamic instability.

\section{Role of the DSMB}

Safety oversight will be under the direction of an independent DSMB. All SAEs will be reported to the site Institutional Review Board (IRB) and MCC within $24 \mathrm{~h}$ of the research team learning about the event followed by a more detailed written report to the local IRB. Sites will inform the MCC of any and all related adverse events (non-serious) within 14 days of discovery. The MCC will prepare summaries of all reports and provide them to the DSMB at least every 6 months. The DSMB will convene by teleconferencing or in person at $25 \%$, $50 \%$ and $75 \%$ of enrolment to review adverse events or earlier if so needed. The NHLBI will be invited to all DSMB meetings and kept apprised of any safety concerns.

\section{Ethics approval}

Approvals of the protocol and informed consent forms were obtained from the DSMB and the NHLBI prior to enrolling the first study participant. In addition, approvals of the protocol and informed consent documents were required and obtained from the IRB of each participating institution prior to enrolling study participants at the respective study site. To ensure that each participating institution's informed consent documentation complied with NHLBI requirements and the code of federal regulations (CFRs) Title 21 Part 50 Section 
50.25, all informed consent forms were reviewed and approved by the MCC. Official documentation of all IRB approvals and all finalised informed consent forms have been collected and stored by the MCC.

\section{Considerations for continuation to a phase III clinical trial}

The decision to proceed with a phase III trial is formally outlined as follows:

1. Initiate phase III study: demonstrated efficacy signal in addition to adequate safety profile.

2. Criteria: primary end point of the trial achieves statistical significance.

3. Further development potentially required: weak efficacy signal.

4. Criteria: primary end point did not achieve a priori level of significance but is in the right direction and there was a general consistency of secondary end points, indicating propensity for efficacy and no increased mortality in the treatment arm.

5. Abandon treatment platform: harm (in efficacy or safety end points).

6. Criteria: study terminated early per recommendation by DSMB for safety and/or risk/benefit ratio concerns (ie, stop for harm, unacceptable risk profile, etc).

\section{Ancillary study}

At the primary centre, all patients enrolled in the study will be invited to join an ancillary study with CT imaging. This substudy will apply established and accepted CT imaging techniques, as well as serial measurement of non-invasive oscillatory impedance of the lung, to seek insights into the mechanism of clinical benefit of the primary trial.

\section{DISCUSSION}

We present the study protocol and data analysis plans for a phase II, multicentre randomised clinical trial that will assess the impact of mechanical ventilation directed at maintaining a positive $\mathrm{P}_{\mathrm{TP}}$ in patients with ARDS. Specifically, we have hypothesised that adjusting ventilator pressure to achieve positive $\mathrm{P}_{\mathrm{TP}}$ values will lead to improvement in a composite outcome of mortality and time off the ventilator at 28 days. Secondarily, this investigation will garner important mechanistic data on $\mathrm{P}_{\mathrm{TP}}$ and its correlation with other surrogate physiological measurements, as well as the potential value of using relevant biomarkers as biochemical surrogate outcome markers for mortality in ARDS. Finally, the results of this study will provide essential information on the scientific merit and feasibility of a larger, phase III trial testing the role of $\mathrm{P}_{\mathrm{TP}}$ to guide ventilation in ARDS.

This trial builds on our previous phase I study by increasing the number of enrolled patients and by increasing the number of participating centres, thereby making the results of the current study more generalisable to the adult acute care hospital population. This strategy will also allow us to demonstrate feasibility of implementing our esophageal pressure management technique across multiple centres.

Another notable strength of the current protocol is a more clinically relevant primary end point as compared with our previous study. Studies of patients admitted to the ICU frequently report ventilator-free days as an outcome. Unfortunately, this measure does not distinguish between patients who have died and those who remained on the ventilator for 28 days. A more clinically useful outcome measure is one that takes mortality into account when calculating VFD. Therefore, we propose a primary hierarchical end point that incorporates death and VFD at 28 days in such a manner that death constitutes a more serious outcome.

Several important limitations with the planned investigation are worth being noted. The first limitation relates to the learning curve among centres in achieving accurate measurements of esophageal pressure. Several of the centres involved in the study routinely use esophageal pressure measurements in clinical practice and are familiar with the technique. Training is provided for all sites, but is particularly important for those sites less familiar with the techniques of esophageal pressure measurement and interpretation. Training includes both didactic lectures in basic respiratory physiology (needed to interpret the physiological waveforms) and hands-on experience. The MCC developed training materials for this purpose. Recurrent training and technical assistance will be available throughout the study. All waveforms will be reviewed by the PCL as a measure of quality assurance as well as to gauge the effectiveness of our educational tools.

A second limitation relates to the time frame of intervention. In the proposed study, the experimental intervention will take place until the patient is weaned from controlled mechanical ventilation or 28 days, after which the clinicians caring for the patient will dictate care. An extended intervention period carries a higher data collection burden, an increased potential for missing data points and a higher difficulty of protocol adherence. However, choosing an extended intervention period allows for a potentially greater separation in mortality curves. Additionally, in clinical practice, most patients are ventilated for less than 28 days. Thus, we anticipate the actual study intervention period to be shorter than 28 days.

A third and final limitation relates to a potential lack of efficacy. The null hypothesis may prove to be correct and there is no benefit to the strategy of maintaining a positive $\mathrm{P}_{\mathrm{TP}}$ during mechanical ventilation. While disappointing, a 'negative' result is an important result. A negative result would be important in informing the community of critical care physicians and suggest a shift of focus to more fruitful therapeutic interventions. A lack of efficacy in the primary outcome may be offset by new findings in the analysis of secondary outcomes, which could guide future research. In addition, the 
physiological and biochemical data generated during the exploration of mechanistic outcomes should lead to important insights into the reasons behind the negative result and generation of important new knowledge.

\section{CONCLUSION}

This manuscript describes the study protocol and analysis plans for the first phase II randomised clinical trial of the use of $\mathrm{P}_{\mathrm{TP}}$ to guide ventilation in patients with ARDS. This protocol represents important improvements from our phase I study by increasing the number of enrolled patients and participating centres, as well as an improved primary end point. In addition to providing important information on the safety and efficacy of the use of esophageal pressure in managing ventilation in patients with moderate-to-severe ARDS, the results of this clinical trial will also inform the scientific community regarding the merit and feasibility of a more definitive phase III clinical trial.

Contributors EF was involved in protocol design, manuscript drafting and revision, and final approval of the submitted version. VN, TS, SL and DT were involved in protocol conception and design, manuscript revision, and final approval of the submitted version. VMB-G was involved in protocol design, manuscript revision and final approval of the submitted version.

Funding This study is supported by the National Heart, Lung, and Blood Institute (grant number UM1-HL108724).

Competing interests None.

Ethics approval Data Safety and Monitoring Board AND Institutional Review Boards for each participating centre.

Provenance and peer review Not commissioned; peer reviewed for ethical and funding approval prior to submission.

Open Access This is an Open Access article distributed in accordance with the Creative Commons Attribution Non Commercial (CC BY-NC 4.0) license, which permits others to distribute, remix, adapt, build upon this work noncommercially, and license their derivative works on different terms, provided the original work is properly cited and the use is non-commercial. See: http:// creativecommons.org/licenses/by-nc/4.0/

\section{REFERENCES}

1. The Acute Respiratory Distress Syndrome Network. Ventilation with lower tidal volumes as compared with traditional tidal volumes for acute lung injury and the acute respiratory distress syndrome. N Engl J Med 2000;342:1301-8.

2. Slutsky AS, Ranieri VM. Mechanical ventilation: lessons from the ARDSNet trial. Respir Res 2000;1:73-7.

3. Briel M, Meade M, Mercat A, et al. Higher vs lower positive end-expiratory pressure in patients with acute lung injury and acute respiratory distress syndrome: systematic review and meta-analysis. JAMA 2010;303:865-73.

4. Gattinoni L, Pelosi P, Suter PM, et al. Acute respiratory distress syndrome caused by pulmonary and extrapulmonary disease. Different syndromes? Am J Respir Crit Care Med 1998;158:3-11.

5. Slutsky AS. Lung injury caused by mechanical ventilation. Chest 1999;116(1 Suppl):9S-15S.

6. Matthay MA, Ware LB, Zimmerman GA. The acute respiratory distress syndrome. J Clin Invest 2012;122:2731-40.

7. Terragni PP, Rosboch GL, Lisi A, et al. How respiratory system mechanics may help in minimising ventilator-induced lung injury in ARDS patients. Eur Respir J Suppl 2003;42:15s-21s.

8. Gattinoni L, Caironi $P$, Carlesso $E$. How to ventilate patients with acute lung injury and acute respiratory distress syndrome. Curr Opin Crit Care 2005;11:69-76.

9. Talmor D, Sarge T, Malhotra A, et al. Mechanical ventilation guided by esophageal pressure in acute lung injury. $N$ Engl J Med 2008;359:2095-104.
10. Ferguson ND, Cook DJ, Guyatt GH, et al. High-frequency oscillation in early acute respiratory distress syndrome. $N$ Engl J Med 2013;368:795-805.

11. Eisner MD, Parsons $P$, Matthay MA, et al. Plasma surfactant protein levels and clinical outcomes in patients with acute lung injury. Thorax 2003;58:983-8.

12. Greene KE, Wright JR, Steinberg KP, et al. Serial changes in surfactant-associated proteins in lung and serum before and after onset of ARDS. Am J Respir Crit Care Med 1999;160:1843-50.

13. Ware LB, Koyama T, Billheimer DD, et al. Prognostic and pathogenetic value of combining clinical and biochemical indices in patients with acute lung injury. Chest 2010;137:288-96.

14. Calfee CS, Ware LB, Eisner MD, et al. Plasma receptor for advanced glycation end products and clinical outcomes in acute lung injury. Thorax 2008;63:1083-9.

15. Christie JD, Shah CV, Kawut SM, et al. Plasma levels of receptor for advanced glycation end products, blood transfusion, and risk of primary graft dysfunction. Am J Respir Crit Care Med 2009;180:1010-15.

16. Fremont RD, Koyama T, Calfee CS, et al. Acute lung injury in patients with traumatic injuries: utility of a panel of biomarkers for diagnosis and pathogenesis. J Trauma 2010;68:1121-7.

17. Agouridakis $P$, Kyriakou D, Alexandrakis MG, et al. The predictive role of serum and bronchoalveolar lavage cytokines and adhesion molecules for acute respiratory distress syndrome development and outcome. Respir Res 2002;3:25.

18. Calfee CS, Eisner MD, Parsons PE, et al. Soluble intercellular adhesion molecule-1 and clinical outcomes in patients with acute lung injury. Intensive Care Med 2009;35:248-57.

19. Covarrubias M, Ware LB, Kawut SM, et al. Plasma intercellular adhesion molecule-1 and von Willebrand factor in primary graft dysfunction after lung transplantation. Am J Transplant 2007;7:2573-8

20. McClintock D, Zhuo $\mathrm{H}$, Wickersham N et al. Biomarkers of inflammation, coagulation and fibrinolysis predict mortality in acute lung injury. Crit Care 2008;12:R41.

21. Bouros D, Alexandrakis MG, Antoniou KM, et al. The clinica significance of serum and bronchoalveolar lavage inflammatory cytokines in patients at risk for acute respiratory distress syndrome. BMC Pulm Med 2004;4:6.

22. Meduri GU, Headley S, Kohler G, et al. Persistent elevation of inflammatory cytokines predicts a poor outcome in ARDS. Plasma IL- 1 beta and IL- 6 levels are consistent and efficient predictors of outcome over time. Chest 1995;107:1062-73.

23. Parsons PE, Eisner MD, Thompson BT, et al. Lower tidal volume ventilation and plasma cytokine markers of inflammation in patients with acute lung injury. Crit Care Med 2005;33:1-6; discussion 230-2.

24. Christie JD, Robinson N, Ware LB, et al. Association of protein $\mathrm{C}$ and type 1 plasminogen activator inhibitor with primary graf dysfunction. Am J Respir Crit Care Med 2007;175:69-74.

25. Prabhakaran $\mathrm{P}$, Ware LB, White KE, et al. Elevated levels of plasminogen activator inhibitor-1 in pulmonary edema fluid are associated with mortality in acute lung injury. Am J Physiol Lung Cell Mol Physiol 2003;285:L20-8.

26. Ware LB, Conner ER, Matthay MA. von Willebrand factor antigen is an independent marker of poor outcome in patients with early acute lung injury. Crit Care Med 2001;29:2325-31.

27. Ware LB, Fang $\mathrm{X}$, Matthay MA. Protein $\mathrm{C}$ and thrombomodulin in human acute lung injury. Am J Physiol Lung Cell Mol Physiol 2003;285:L514-21.

28. Ware LB, Matthay MA, Parsons PE, et al. Pathogenetic and prognostic significance of altered coagulation and fibrinolysis in acute lung injury/acute respiratory distress syndrome. Crit Care Med 2007;35:1821-8.

29. Rubin DB, Wiener-Kronish JP, Murray JF, et al. Elevated von Willebrand factor antigen is an early plasma predictor of acute lung injury in nonpulmonary sepsis syndrome. J Clin Invest 1990;86:474-80.

30. Ware LB, Eisner MD, Thompson BT, et al. Significance of von Willebrand factor in septic and nonseptic patients with acute lung injury. Am J Respir Crit Care Med 2004;170:766-72.

31. Crotti S, Mascheroni D, Caironi $P$, et al. Recruitment and derecruitment during acute respiratory failure: a clinical study. $A m \mathrm{~J}$ Respir Crit Care Med 2001;164:131-40.

32. Ranieri VM, Suter PM, Tortorella C et al. Effect of mechanical ventilation on inflammatory mediators in patients with acute respiratory distress syndrome: a randomized controlled trial. JAMA 1999;282:54-61.

33. Parsons PE, Matthay MA, Ware LB, et al. Elevated plasma levels of soluble TNF receptors are associated with morbidity and mortality in patients with acute lung injury. Am J Physiol Lung Cell Mol Physiol 2005;288:L426-31 